\title{
From nonequilibrium single-molecules trajectories to underlying dynamics
}

Alexander M. Berezhkovskii' and Dmitrii E. Makarov ${ }^{23}$

'Mathematical and Statistical Computing Laboratory, Office of Intramural Research, Center for

Information Technology, National Institutes of Health, Bethesda, Maryland 20892, USA

2Department of Chemistry, University of Texas at Austin, Austin, Texas 78712

${ }^{3}$ Oden Institute for Computational Engineering and Sciences, University of Texas at Austin, Austin, Texas 78712 


\section{Derivation of Eq. 16.}

Consider a particle injected at $x=0$. Under the assumption of diffusive dynamics in a potential $U(x)$, we would like to know the mean time $\langle\tau\rangle$ that the particle will spend inside the interval $(a, b), a<0<b$, before it escapes this interval. Note that in Eq. $16 b=-a=L$, where $L$ is the length of the periodic cell. To answer this question we imagine a steady-state scenario in which a flux $j$ of particles is injected at $x=0$. These particles eventually escape either through the left boundary $a$ (with a corresponding flux $j_{a}$ ) or through the right boundary $b$ (with a corresponding flux $j_{b}$ ), such that we have $j=j_{a}+j_{b}$.

We note the following relation

$$
j_{a(b)}=j \Phi(0 \rightarrow a(b) \mid b(a)) \equiv j \Phi_{a(b)},
$$

where $\Phi_{a(b)}$ is the splitting probability that the particle originating from $x=0$ crosses the boundary $a(b)$ before crossing the other boundary.

Now consider the particles in the interval $(a<x<0)$. Their flux is the same anywhere in the interval and is given by the usual expression for the flux in the system described by the Smoluchowski equation:

$$
j_{a}=D(x) e^{-\beta U(x)} \frac{\partial}{\partial x} e^{\beta U(x)} c_{a}(x),(\mathrm{S} 1)
$$

where $c_{a}(x)$ is the concentration of particles at point $x, a<x<0$. The sign convention here is such that the flux of particles toward the left boundary is positive. Since the particles are absorbed at 
the boundary, we must impose the absorbing boundary condition, $c_{a}(a)=0$, after which $c_{a}(x)$ can be determined from Eq. S1:

$c_{a}(x)=j_{a} e^{-\beta U(x)} \int_{a}^{x} e^{\beta U(y)} \frac{d y}{D(y)}(\mathrm{S} 2)$

Similarly, we find the concentration profile for the particles in the interval $0<x<b$,

$c_{b}(x)=j_{b} e^{-\beta U(x)} \int_{x}^{b} e^{\beta U(y)} \frac{d y}{D(y)},(\mathrm{S} 3)$

where the sign convention is such that the flux of particles moving from left to right is positive.

Since Eqs. S2 and S3 should give the same result at $x=0, c_{a}(0)=c_{b}(0)$, we immediately obtain the ratio for the splitting probabilities,

$\frac{j_{b}}{j_{a}}=\frac{\Phi_{b}}{\Phi_{a}}=\frac{\int_{x}^{b} e^{\beta U(y)} \frac{d y}{D(y)}}{\int_{a}^{x} e^{\beta U(y)} \frac{d y}{D(y)}},(\mathrm{S} 4)$

which, combined with the condition $\Phi_{a}+\Phi_{b}=1$, gives the known result (cf. Eq. 10 in the main text):

$\Phi_{b}=1-\Phi_{a}=\frac{\int_{x}^{b} e^{\beta U(y)} \frac{d y}{D(y)}}{\int_{a}^{b} e^{\beta U(y)} \frac{d y}{D(y)}}(\mathrm{S} 5)$

The lifetime $\langle\tau\rangle$ can now be determined from the intuitively appealing relationship (which is in fact exact) 
$\frac{N_{s s}}{\langle\tau\rangle}=j(\mathrm{~S} 6)$

between the injected (and escaping) flux $j$ and the steady-state value of the total number of particles present in the interval $(a, b)$. The latter is given by

$$
N_{s s}=\int_{a}^{0} c_{a}(x) d x+\int_{0}^{b} c_{b}(x) d x(\mathrm{~S} 7)
$$

Combining Eqs. S3-S7, we find

$$
\langle\tau\rangle=\Phi_{a} \int_{a}^{0} d x e^{-\beta U(x)}\left(\int_{a}^{x} e^{\beta U(y)} \frac{d y}{D(y)}\right)+\Phi_{b} \int_{0}^{b} d x e^{-\beta U(x)}\left(\int_{x}^{b} e^{\beta U(y)} \frac{d y}{D(y)}\right),
$$

or, using Eq. S5,

$\langle\tau\rangle=\left(\int_{a}^{b} e^{\beta U(y)} \frac{d y}{D(y)}\right)\left\{\Phi_{a} \int_{a}^{0} d x e^{-\beta U(x)} \Phi(x \rightarrow b \mid a)+\Phi_{b} \int_{0}^{b} d x e^{-\beta U(x)} \Phi(x \rightarrow a \mid b)\right\}(\mathrm{S} 9)$

If the diffusivity is coordinate independent, $D(x)=D$, then from Eq. S5 we have:

$e^{-\beta U(x)}=\frac{1}{\left(\int_{a}^{b} e^{\beta U(y)} \frac{d y}{D(y)}\right) \frac{d \Phi(x \rightarrow b \mid a)}{d x}}(\mathrm{~S} 10)$.

Substituting this into Eq. S9 we finally obtain

$$
\langle\tau\rangle=\frac{1}{D}\left[\Phi_{a} \int_{a}^{0} \Phi(x \rightarrow b \mid a) \frac{d x}{d \Phi(x \rightarrow b \mid a) / d x}+\Phi_{b} \int_{0}^{b} \Phi(x \rightarrow a \mid b) \frac{d x}{d \Phi(x \rightarrow a \mid b) / d x}\right]
$$

Eq. S11 is equivalent to Eq. 16 in the main text when $a=-L$ and $b=+L$. 


\section{Comparison of methods for estimating effective potentials from nonequilibrium trajectories.}

A family of methods proposed in various contexts start with the Smoluchowski equation for the steady-state flux

$j_{s s}=-\beta D U^{\prime}(x) \rho_{s s}(x)-D \rho_{s s}^{\prime}(x),(\mathrm{S} 12)$

where $\rho_{s s}(x)$ is the steady-state distribution of the coordinate $x$. This allows one to obtain the following expression for the derivative of the potential,

$u(x) \equiv-\beta D U^{\prime}(x)=\frac{j_{s s}}{\rho_{s s}(x)}+D \frac{d \ln \rho_{s s}(x)}{d x}$

This result is superficially similar to our Eqs.6-7 written for the local drift velocity, especially after one defines a velocity $v(x)=j_{s s} / \rho_{s s}(x)$ associated with the steady-state flux, as in ref... The two approaches are however different because $v(x)$ and the drift velocity $u(x)$ are different quantities. Indeed, Eqs.6-7 do not contain the second term appearing in Eq. S13. Moreover, the drift velocity $u(x)$ is nonzero regardless of whether or not the system is in equilibrium. In contrast, when Eq. S13 is applied to the equilibrium case the velocity $v(x)$ vanishes since there is no flux in the system. The equilibrium distribution then arises entirely from the second term in Eq. S13. Eqs. 6-7, on the other hand, are similar to the trajectory analysis proposed by the de Oliveira group for equilirium systems ${ }^{23}$.

The specific steady state used in Eq. S13 depends on the boundary conditions. Zhang et al ${ }^{4}$ and Berkovich et al' consider a streching/relaxation cycle, where the system, once achieving a specified extension value, $x=x_{2}$, is re-stretched to the initial extension $x=x_{1}$. This implies an 
absorbing boudary condition at $x_{2}$ with the steady-state probability flux reinjected at $x=x_{1}$ (and where no boundary condition is imposed at $x_{2}$ ). In contrast, the work on molecular motors usually deals with periodic boundary conditions $\mathbf{s}^{5.8}$, where the dynamics of the motor is mapped onto one or more periods, similarly to the work presented here. It is important to keep in mind that the steady-state flux and the steady-state probability distribution appearing in Eq. S.13 depend on the boudary conditions used.

In general, the family of methods that are based on Eq. S13 require an independent estimate of the diffusivity $D$, which is a difficult task unless its value is dominated by the hydrodynamic drag on the probe ${ }^{7}$. The stretching/relaxation scenario, however, allows one to obtain an estimate of the potential $U(x)$ that is independent of the diffusivity ${ }^{4}$ To see this, we notice that, because of the boundary condition at $x=x_{2}\left(\rho_{s s}\left(x_{2}\right)=0\right)$, Eq. S12 reduces to $j_{s s}=-D \rho_{s s}^{\prime}\left(x_{2}\right)$. When this expression for the steady-state flux is subsituted into Eq. S13, the diffusivity $D$ can be eliminated from the final result, albeit at the expense of having to compute a derivative of the probability density $\rho_{s s}(x)$ near a point where it is equal to zero and thus is poorly sampled. Independence of the value of diffusivity (at least when it is assumed constant) is a feature that the method of Zhang et $\mathrm{al}^{4}$ shares with the approach based on the splitting probabilities discussed here. We note that, although the boundary conditions in the stretching/relaxation scenario are diferent from those in molecular motor studies, it is possible to partition a single-molecule trajectory of a motor into segments that can be used to form an ensemble of trajectories for the stretching/relaxation scenario. 
(1) Berkovich, R.; Fernandez, V. I.; Stirnemann, G.; Valle-Orero, J.; Fernandez, J. M.

Segmentation and the Entropic Elasticity of Modular Proteins. J Phys Chem Lett 2018, 9, 47074713.

(2) de Oliveira, R. J. Stochastic diffusion framework determines the free-energy landscape and rate from single-molecule trajectory. J. Chem. Phys. 2018, 149, 234107.

(3) Freitas, F. C.; Lima, A. N.; Contessoto, V. G.; Whitford, P. C.; Oliveira, R. J. Driftdiffusion (DrDiff) framework determines kinetics and thermodynamics of two-state folding trajectory and tunes diffusion models. J. Chem. Phys. 2019, 151, 114106.

(4) Zhang, Q.; Brujic, J.; Vanden-Eijnden, E. Reconstructing Free Energy Profiles from Nonequilibrium Relaxation Trajectories. Journal of Statistical Physics 2011, 144, 344-366. (5) Wang, H. Several Issues in Modeling Molecular Motors. Journal of Computational and Theoretical Nanoscience 2008, 5, 2311-2345.

(6) Lopez-Alamilla, N. J.; Jack, M. W.; Challis, K. J. Reconstructing free-energy landscapes for cyclic molecular motors using full multidimensional or partial one-dimensional dynamic information. Phys Rev E 2019, 100, 012404.

(7) Lopez-Alamilla, N. J.; Jack, M. W.; Challis, K. J. Analysing single-molecule trajectories to reconstruct free-energy landscapes of cyclic motor proteins. J. Theor. Biol. 2019, 462, 321328.

(8) Reimann, P. Brownian motors: noisy transport far from equilibrium. Physics Reports 2002, 361, 57-265. 